\title{
GOBERNANZA EN EL ANÁLISIS DEL BIENESTAR SOCIAL
}

\section{GOVERNANCE IN THE ANALYSIS OF SOCIAL WELFARE}

\author{
Guillermo Calderón-Ramírez ${ }^{1}$ \\ Universidad Nacional de Costa Rica, Costa Rica
}

\begin{abstract}
RESUMEN
El tema de la pobreza es de gran importancia mundial, pactado en todas las Naciones como uno de los principales objetivos a cumplir. Se destaca en este documento algunos conceptos claves para el estudio de la pobreza desde una perspectiva de la geografía política, área de gran importancia para los geógrafos. Se realiza una síntesis bibliográfica de temas que buscan explicar la evaluación de políticas sociales. La intención es explicar cómo los conceptos de política, Estado-Nación, política pública, política social y región llevan a un profundo análisis de la pobreza y gobernanza de esta. Al experimentar distintas concepciones de pobreza y medición de la misma se parte con la idea de definir por medio de la gobernanza una serie de posibles términos que en su conjunto puedan favorecen la operacionalidad de la pobreza. Se han considerado variables definidas por el Programa de las Naciones Unidas para el desarrollo como lo son la salud, educación, renta y vivienda, encontrando una descentralización de las política públicas en Costa Rica, país de estudio de caso, el cual se toma como referencia debido a que es referente en temas de salud y economía a nivel centroamericano.
\end{abstract}

Palabras clave: pobreza, gobernanza, política, región.

\begin{abstract}
The issue of poverty is of global importance, it has been agreed in all nations as one of the main objectives to fulfill. This paper highlights some key concepts for studying poverty from the

1 Máster en Geografía Política. Académico e investigador, Escuela de Ciencias Geográficas, Universidad Nacional de Costa Rica, Costa Rica. Correo electrónico: gcalderon2809@gmail.com
\end{abstract}

Fecha de recepción: 25 de agosto de 2016

Fecha de aceptación: 18 de octubre de 2016 
perspective of political geography -area of great importance for geographers. The intention is to explain how the concepts of politics, nation-state, public politics-social politics and regional policy lead to a thorough analysis of poverty and its governance. By experiencing different conceptions of poverty and measuring there is the idea of defining governance through a number of possible terms that together can favor the operationalization of poverty. Because of the aforementioned, this article intends to build a conceptual foundation for the study of poverty and its governance.

Keywords: poverty, governance, politics, region.

\section{Introducción}

El tema del bienestar social analizado desde la pobreza trae consigo una serie de cuestionamientos, esto debido a que se tiene distintas interpretaciones sobre el concepto de pobreza y la manera de cómo se mide la misma. Por lo anterior, se trae en el presente artículo un acercamiento de conceptos claves para evaluar la pobreza y su posterior gobernanza. Además se ejemplifica con el caso de políticas públicas en función del bienestar social costarricense. Conceptos como política, Estado, fundamentación de que es pobreza, políticas públicas a una escala regional son las llaves para tal concepción.

El hablar de pobreza por si solo ya trae consigo una serie de incertidumbre, y abonado a las políticas públicas o su gobernanza se torna posiblemente difícil de discutir. Parafraseando a Abranches (1998) menciona que es frecuente que las políticas que tienen como fin combatir la pobreza tengan efectos negativos, debido a que se podría estar trabajando en necesidades diferentes, es decir posible descentralización.

La pobreza alcanza todo orden social y económico, siendo prudente comprender los conflictos internos de la sociedad dentro de un determinado territorio. Es necesario puntualizar que el abordaje de este artículo se referencia a la geografía política, en el que las políticas públicas contribuyen para disminuir la pobreza, pero que no son el foco central para la disminución de la misma. En fin el propósito es introducir al lector en el tema de la pobreza y la política pública, con la intensión de entender como estas se relacionan en el accionar del Estado.

\section{Metodología}

Se genera una revisión bibliográfica sobre conceptos que podrían verse relacionados con el tema social, puntualmente con la pobreza. Se discute la percepción de las distintas concepciones planteadas, para 
brindarle al lector una visión acerca del posible tratamiento en el estudio del tema principal que se discute. Posteriormente a manera de ejemplo se recaba información que caracteriza el tratamiento de las políticas públicas relacionadas con la pobreza costarricense, en un período de los últimos 30 años aproximadamente.

\section{Resultados}

\section{Pobreza según contexto de estudio}

La población en condición de pobreza presenta no solo un déficit en condiciones económicas sino también impotencia con respecto al acceso político, al no tener el poderío económico pasa desapercibido en las decisiones políticas, por ende la importancia del Estado como mediador de clases socioeconómicas. La acción para disminuir la pobreza no solo se basa en términos económicos, fusiona todo un conjunto de variables e intereses que deben desarrollarse paralelamente. La política es una de ellas tiene el poder de buscar el bien común dentro de su Nación, en donde los sectores sociales, económicos y por ende políticos logren sintetizar y conllevar un tema que debe ser de interés nacional, siendo que:

...um indivíduo não é mais pobre ou menos pobre porque consome um pouco menos ou um pouco mais. A definição de pobreza deve ir além dessa pesquisa estatística para situar o homem na sociedade global à qual pertence, porquanto a pobreza não é apenas uma categoria econômica, mas também uma categoria política acima de tudo. Estamos lidando com um problema social. (Santos, 1978, p.10).

Se debe disminuir la pobreza y lograr una igualdad tanto económica como social, sin distinción de raza, color o etnia, en donde este individuo de la sociedad puede desenvolverse a fin de su propio ambiente, Freire (1968) por medio de Santos (1978) menciona que la pobreza no es un problema que deba colocar a la población pobre a la altura del opresor con la intención de que se parezca más a este, y sí que se transforme la estructura logrando que cada individuo se desenvuelva conforme a lo que es.

La pobreza interioriza las clases sociales a partir de circuitos teniendo el circuito superior en el que se interrelaciona el comercio, la bancas e industria; y el circuito inferior conjugando el comercio de pequeña escala, 
la producción por medio de capital no intensivo, y servicios no modernos, (Santos, 1978). La clave en el progreso de las naciones es la intervención política, es por ello que se asocia la pobreza con las políticas públicas ya que esta es una de las maneras más bondadosas de la sociedad porque busca el bien común de una Nación.

Pobreza está asociada al poco desarrollo socioeconómico y las características de los pobres varían según país. Por lo tanto disminuir la pobreza debe ser de interes de todo Estado ya que la situación de los pobres "não melhoram porque as oportunidades para fazê-lo são menos acessíveis a eles, pobres, e porque não lhes sobra tempo e espaço para acumular, ainda que gratuitamente, os recursos necessários para alcançar melhores condições de vida." (Abranches, 1998, p.17). Los pobres consumen su tiempo laborando períodos mayores, dejando de lado dedicación para la familia, educación u ocio. Se debe tener presente que la medición de la pobreza va más allá de la medida de ingresos, Artigas (2003) se refiere a que en términos de desarrollo humano la pobreza es mucho más amplia a la posible falta de ingresos, asociándola con pobreza humana.

La problemática es entender la pobreza en sus distintas concepciones basado en el funcionamiento de esta y cuáles son sus principales fuentes reproductoras, descubriendo la manera en que podría ser atendida consiguiendo reducir un panorama poco favorable para Latinoamérica en el que su pobreza para el año 2012 ronda el 28\% de su población según la CEPAL.

La relación que se evalúa a partir del IDH con las políticas públicas parte como elemento para diagnosticar la situación de la pobreza. Es claro que las variables estudiadas (vivienda, salud, educación y renta) no se pueden dejar de lado una de otra, o estudiar por separado por lo menos en esta investigación se trae dicha discusión, ya que el acceso a la salud o la esperanza de vida al nacer traerá posteriores beneficios en la formación educacional y posible desempeño en el empleo. Lo anterior se puede resumir en un círculo donde la salud esta como primera instancia siendo fuente pragmática para la educación y esta última podrá llevar a niveles de empleo estables y con remuneración equilibrada según su calificación profesional.

\section{Política mediadora del Estado-Nación}

Algunos científicos políticos como Trigal y Pozo (1999) mencionan que el realizar evaluaciones en la política está podría ser llamada de 
herramienta privilegiada de una Nación, debido a que obliga a los responsables a tomar medidas sobre las decisiones en la formulación y ejecución de las políticas.

La capacidad que tiene la política de regular la sociedad, menciona Wolff (2003) se define por dos aspectos en primer lugar necesita de una comunidad y esta a su vez requiere poder. La existencia de la política se da a partir del momento en que una comunidad coloca la interrogante de "poder" o en el instante que un "poder" ejercido por algunos se practica en una comunidad considerando el modo de vida.

La política “...trata de convivência entre diferentes.” (Arendt, 2003, p.21). Dicha interacción entre sociedad y política Castro (2005) destaca que en toda sociedad existirá diferentes intereses, y que por consiguiente la realización de los mismos genera conflictos, por ende se parte de la política para poder organizar esos conflictos, en donde la población puede alcanzar sus objetivos, reflejando que la política no se puede dejar de lado siendo el núcleo de organización de sociedades complejas.

Además presenta una estrecha relación en la visión de la geografía, apunta Castro (2005) que algunas acciones como la globalización, el debilitamiento del Estado-Nación, mayor control de las fronteras, la expansión significativa de la democracia, intensificación de la pobreza junto a un fortalecimiento de los movimientos sociales y de los derechos ciudadanos, presentan una importancia en la geografía política, junto a la aceptación de sus temas y de la congruencia de sus aportes a las nuevas interrogantes dentro del contexto actual. Por tanto se entiende la geografía política según misma autora "como um conjunto de idéias políticas e acadêmicas sobre as relações da geografia com a política e vice-versa." (p.17).

La relación existente entre Estado y política es inseparable Novaes (2003) indica que "o Estado se superpõe deliberadamente à política, e a "política", deliberadamente, inclina-se a servi-lo." (p.16). La política es una manera de organización, siendo la misma concreta en la cual los agentes involucrados en la formulación de la misma están en busca de una homogeneidad de intereses, teniendo la capacidad de organizar los Estados más al mismo tiempo tiene las herramientas para dirigir las Naciones. Es un instrumento capaz de regular la sociedad, ya que al imponer pautas donde existen diferentes intereses del ser humano, la política debe ser acatada, buscando el bien común. 
En el papel que desempeña el Estado en el contexto de la política, Mann (1992) a través de Castro (2005) señala que la particularidad del Estado en comparación a otras formas de organización política se basa en su poder de centralidad territorial definiendo normas y logrando ejecutar las mismas sobre la sociedad dentro de su territorio.

\section{Estado-Nación y su influencia territorial}

La investigación tiene un interés particular entre Estado y Nación o Estado-Nación y la relación de las mismas en la geografía política. “...el Estado será uno de los argumentos centrales, si no el central, de la geografía política que nacerá a finales del siglo XIX.” (Font; Rufí, 2001, p.33). Además Ratzel trabaja con la idea de estudiar el Estado con mayor exactitud en una geografía política, teniendo la idea de explicar científicamente cómo se comporta el Estado territorialmente.

Se argumenta que el Estado es considerado como un sistema de administración de su Nación, el cual desarrolla distintas doctrinas políticas, "o Estado como sistema político é, com respeito ao sistema social um subsistema." (Bobbio, 1987, p.62). En cuanto que para Novaes (2003) lo describe como la manera visible de la soberanía, con lo cual pasa a tener la autoridad de resolver con la violencia, en caso de que la ideología falle, cualquier conflicto que emerja en la sociedad. La sociedad y sus componentes económicos, políticos y territoriales han sido estructurados en Estados, (Font; Rufí, 2001).

Una perspectiva del Estado con visión geográfica Johnston (1982) por medio de Font y Rufí (2001) menciona que el Estado desempeña seis funciones, la primera describe el Estado como protector de su población en el cual existe soberanía, desarrollando una defensa y seguridad hacia las agresiones exteriores. La segunda función es de arbitraje con un organismo legislativo en donde su sistema tiene la finalidad de resolver conflictos entre su población e intereses. La tercera es la fuerza de cohesión, la cual debe contener su propia legitimidad para la cohesión, es decir ser creíble.

Como cuarta función le es denominada de facilitador, siendo el ente que crea y regula las condiciones para la producción económica. Como quinto elemento el Estado es considerado como inversor, desempeñando la función de inversionista financiando investigaciones o aún mejor contribuyendo con la educación. La sexta función considerada de burocracia 
es encargada de que el resto de las funciones puedan desarrollarse adecuadamente, es decir catalogada como la "máquina" del Estado. La Nación es definida según Valéry y Renan a través de Novaes (2003) como un espacio del territorio, con un tiempo de la historia nacional y una cultura homogénea la cual contempla toda la población.

La unión de Estado-Nación, Johnston et al. (2000) la define como la complejidad de instituciones que son llevadas a cabo en el gobierno sobre un determinado territorio. Además es importante resaltar que el EstadoNación tiene su fundamento geográfico refiriéndose a que "permanece antes de tudo como uma unidade política espacial." (Britto, 1986, p.20), es por ello que se resalta la importancia de la geografía en los Estados-Nacionales con poder político. Las características del Estado es ser regulador de la Nación, siendo conformado por el pueblo y el territorio en el que se ejecutan las políticas, influenciado por el poder que el Estado ejerce.

La relación existente entre Estado-Nación tiene sus inicio en los hogares clasificados como unidades reproductoras de la sociedad, esta conformación se torna en una Nación consolidada en la cual se interrelacionan aspectos sociales, económicos y políticos. A partir de allí esta Nación es dividida según condición socioeconómica (clase) y en el otro extremo la Nación es el que habilita el sistema interestatal es decir al Estado, (figura 1). 
Figura 1. Los vínculos institucionales fundamentales

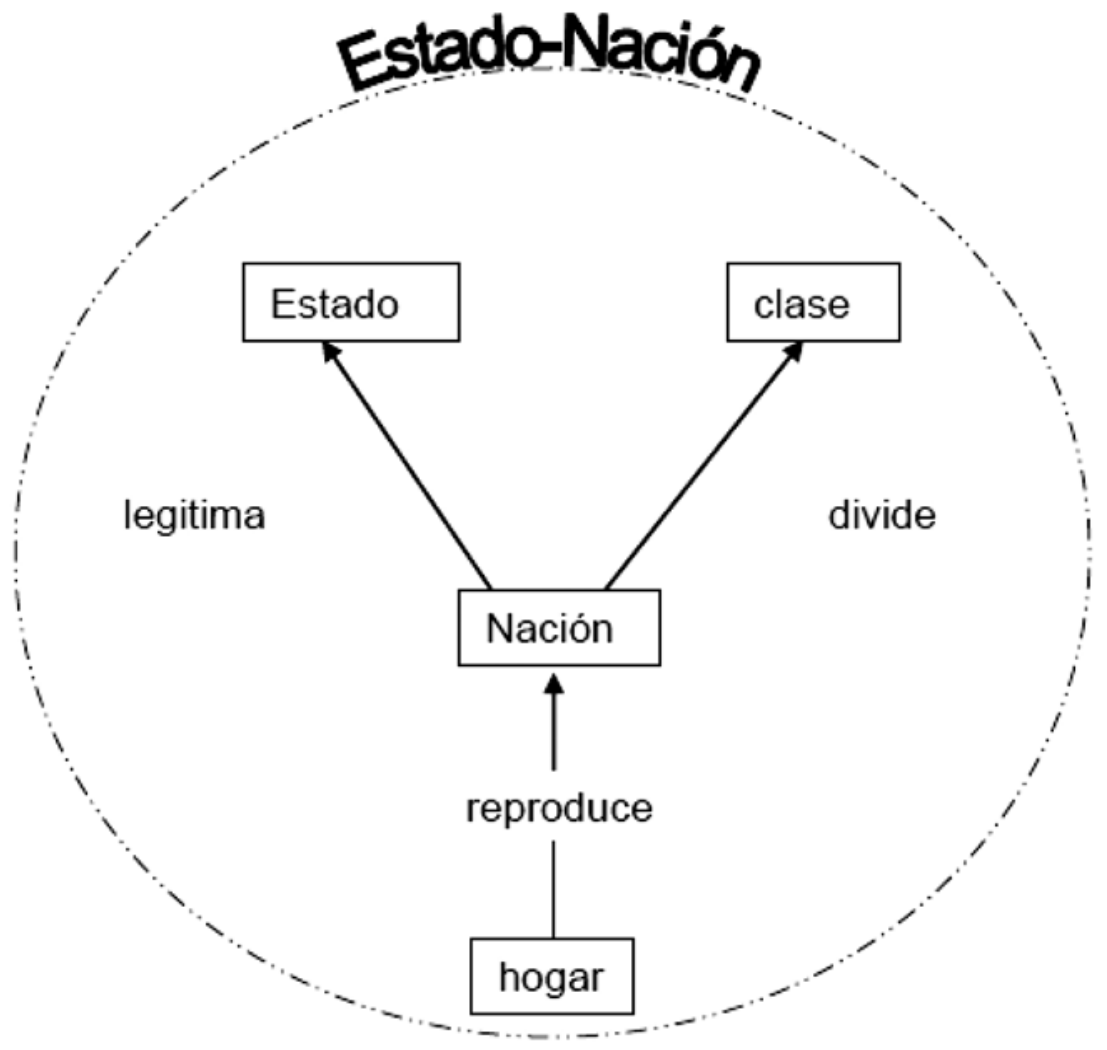

Fuente: Taylor y Flint (2002), p.34.

La relevancia entre Estado y Nación es su estrecha relación, en la que existe una subdivisión de elementos como lo son los hogares y las clases. Esta relación de cuatro instituciones que Taylor y Flint desarrollan, consigue explicar el inicio de los Estado-Nación, pero falta mencionar como esa interacción institucional es desarrollada en un territorio, y conforme a los distintos intereses posee su propia caracterización. Con todo esto en la presentación del modelo anterior se desea elevar la importancia espacial en las investigaciones geográficas.

Como parte de la complejidad del tema desarrollado se describe de manera desagregada los distintos conceptos que intervienen directamente en la política, teniendo su explicación teórica. A partir de la descripción de conceptos como política, política pública, política social, Estado-Nación, 
llegan a interconectarse logrando desenvolverse y permitirse intervenir en el desarrollo socioeconómico nacional o regional.

\section{Políticas públicas-políticas sociales}

El hablar de política pública en un plano general y abstracto debe contener las estructuras de poder y de dominación, y posteriores conflictos a los que la sociedad se enfrenta y encuentra en el Estado un lugar para ser administrado. En un contexto concreto el concepto de política pública debe considerar los recursos de poder que tienen como referente al Estado, principalmente su eje gubernamental, (Azevedo, 1997).

El tema de las políticas públicas es de relevancia, con lo cual en este trabajo se toma como fuente de análisis y se relaciona con la pobreza, siendo el Estado el que regula las mismas dando soluciones a los problemas sociales. El nacimiento de política pública como área de conocimiento y campo académico se debe a los Estados Unidos, dejando de lado los procesos seguidos por los europeos los cuales se centraban en el análisis del Estado y sus instituciones, y no en la producción del gobierno (Souza, 2006). Es decir dicha área surge sin las relaciones teóricas sobre el papel del Estado, pasando directo a estudiar las acciones de los gobiernos.

Es pertinente destacar que no existe una única definición para el concepto de política pública y Souza (2006) deja claro eso al parafrasear a Mead (1995) en donde "a define como un campo dentro do estudo da política que analiza o governo à luz de grandes questões públicas" (Souza, 2006, p.24) pero además misma autora se refiere a Lynn (1980) destacando que este define política pública "como um conjunto de ações do governo que irão produzir efeitos específicos” (p.24).

La definición que logra resumir misma autora con respecto a política pública se considera para esta investigación como la más atenuante y relevante diciendo que es "...o campo do conhecimento que busca, ao mesmo tempo colocar o governo em ação e/ou analisar essa ação (variável independente) e, quando necessário, propor mudanças no rumo ou curso dessas ações (variável dependente)." (p.26). Ahora cabe indicar que la política pública presenta un abordaje abstracto de la política social, en donde la política social puede considerarse "do ponto de vista do Estado, como proposta planeada de enfrentamento das desigualdades sociais" (Demo, 1996, p.14). 
En la política social se tiene que saber cuál es la población objetivo, el desarrollo de estas debe direccionarse con mayor fuerza a atender a la población que se encuentra en pobreza. Es decir existe una proporción poblacional que se denominaría el "dominante" o la que contiene la riqueza y otra porción que pasaría a ser el "dominado" que trabajaría para proporcionar esa riqueza, teniendo las políticas sociales un mayor vigor para la población dominada. Es decir que las políticas sociales deben ser congruentes conforme a las características de su población, tomando en cuenta aspectos económicos, sociales, culturales y políticos.

\section{Región como área de estudio}

La intensión de discutir el concepto de región se debe a dimensión del recorte espacial. La investigación se acentúa en las seis regiones socioeconómicas de Costa Rica, siendo que cada una de ellas presenta características diferenciadas, siendo relevante en el estudio geográfico. Con esto se menciona que para aquellos que conciben la geografía regional como ciencia espacial consideran a la región como "una de las formas más lógicas y satisfactorias de organizar la información geográfica." (Haggett, et al. 1977 apud Johnston, et al. 2000, p. 483).

Las regiones socioeconómicas de Costa Rica presentan una influencia en el desarrollo tanto a nivel económico, social y político. La región tiene un fuerte sentido de unidad administrativa "a divisão regional é o meio pelo qual se exerce frequentemente a hierarquia e o controle na administração dos Estados." (Gomes, 2000, p.53). Entendiendo región como unidad administrativa es clave importante en el contexto del desarrollo socioeconómico costarricense. Las regiones aquí discutidas presentan características estrictamente diferenciadas por un lado la región Central con una influencia urbano-industrial y por otro lado la región Huetar Norte con mayor predominio agropecuario.

Dividir el espacio en regiones presenta la función de establecer de acuerdo a características homogéneas de ciertas áreas una integración del espacio, es decir a través de esas caracterización se logra integrar una serie de variables tanto políticas, sociales, ambientales y económicas, dejando de ser un espacio continuo y que particularmente interesa para el desarrollo de la misma. 
Regionalizar puede ser utilizada como una herramienta en investigaciones geográficos es, “...um meio para a demonstração de uma hipótese e não mais um produto final do trabalho de pesquisa." (Gomes, 2000, p.63). Este autor indica que las regiones envuelven una dinámica propia, desde una visión regional partiendo de la geografía, la cual envuelve la función de caracterizar las unidades regionales en la distribución espacial de los fenómenos. Afirma que el concepto de región tiene implicaciones en el campo de la política, dinámica del Estado, organización cultural y del estatuto de la diversidad espacial.

El regionalizar proporciona una fuente de análisis única, las características que la distingue hacen que sea considerada como “...uma importante categoria de análise, importante para que se possa captar a maneira como uma mesma forma de produzir se realiza em partes específicas do Planeta ou dentro de um país, associando a nova dinâmica as condições preexistentes". (Santos, 1988, p.47).

Al hablar de región con fundamento geográfico no se puede entrar en el equívoco de concebirla como región natural, para esto Gomes (2000) señala que con base a la perspectiva posibilista las regiones tienen existencia como unidades básicas del conocimiento geográfico y no como unidad morfológica. Es decir es la civilización, la interacción humana la que debe ser estudiada para comprender una región. Afirma que el método regional o en la distribución espacial la caracterización de unidades regionales es lo que hace a la geografía ser particularmente diferente a las demás ciencias, es decir el interés por estudiar la distribución y la localización espacial.

La región indica Lencioni (2003) es un espacio que posee características tanto físicas como socioculturales homogéneas, la cual tiene toda una historia que hacen que el hombre se relacione con el territorio, teniendo la particularidad de ser propia. "Integração e sínteses passaram a se constituir na nova motivação do pensamento geográfico. Integração... tanto naturais como sociais, e sínteses da natureza com os elementos socioculturais..." (p.100). El utilizar la región como herramienta de análisis en la geografía proporciona información que puede ser estrictamente comparada con otras regiones. Posee un dominio de características que la distinguen de las demás y no necesariamente debe presentar siempre los mismos criterios de relación, siendo que la región es una "área sob um certo domínio ou área definida por uma regularidade de propriedades que a definem." (Gomes, 2000, p.54), pero si se llega "na 
medida em que os critérios de classificação e divisão do espaço são uniformes, só interessa neste espaço aquilo que é geral..." (p.63), tornando una homogenización dentro de la región definida.

El realizar investigaciones a escala regional representa una mayor integración del Estado-Nación, es decir se concluye que el desarrollo desigual denominado en este trabajo como socioeconómico “...obliga inevitablemente al Estado a organizar su territorio por medio de alguna autonomía local." (Taylor y Flint, 2000, p.358), el distinguir un territorio por sus características homogéneas hacen que esta contenga su propio sistema de desarrollo pero siempre y cuando se respeten las pautas del gobierno central. Se cree que el gobierno central a partir de su organización regional o inclusive local logra intervenir con mayor fuerza en las distintas necesidades sociales, económicas y políticas que puedan surgir en una determinada región, ya que su intervención sería de manera directa, identificando la falencia en un determinado territorio que presenta una serie de características homogéneas.

\section{Caracterización de las políticas públicas en Costa Rica a través de los programas sociales}

Para conocer la manera el desenvolvimiento de las políticas públicas en relación al bienestar social a continuación se ejemplifica la situación en Costa Rica según cuatro variables en función de la vivienda, salud, educación y renta desde antes de los años 1990 hasta el 2010. 
Figura 2. Hogares pobres en Costa Rica, 2000-2010

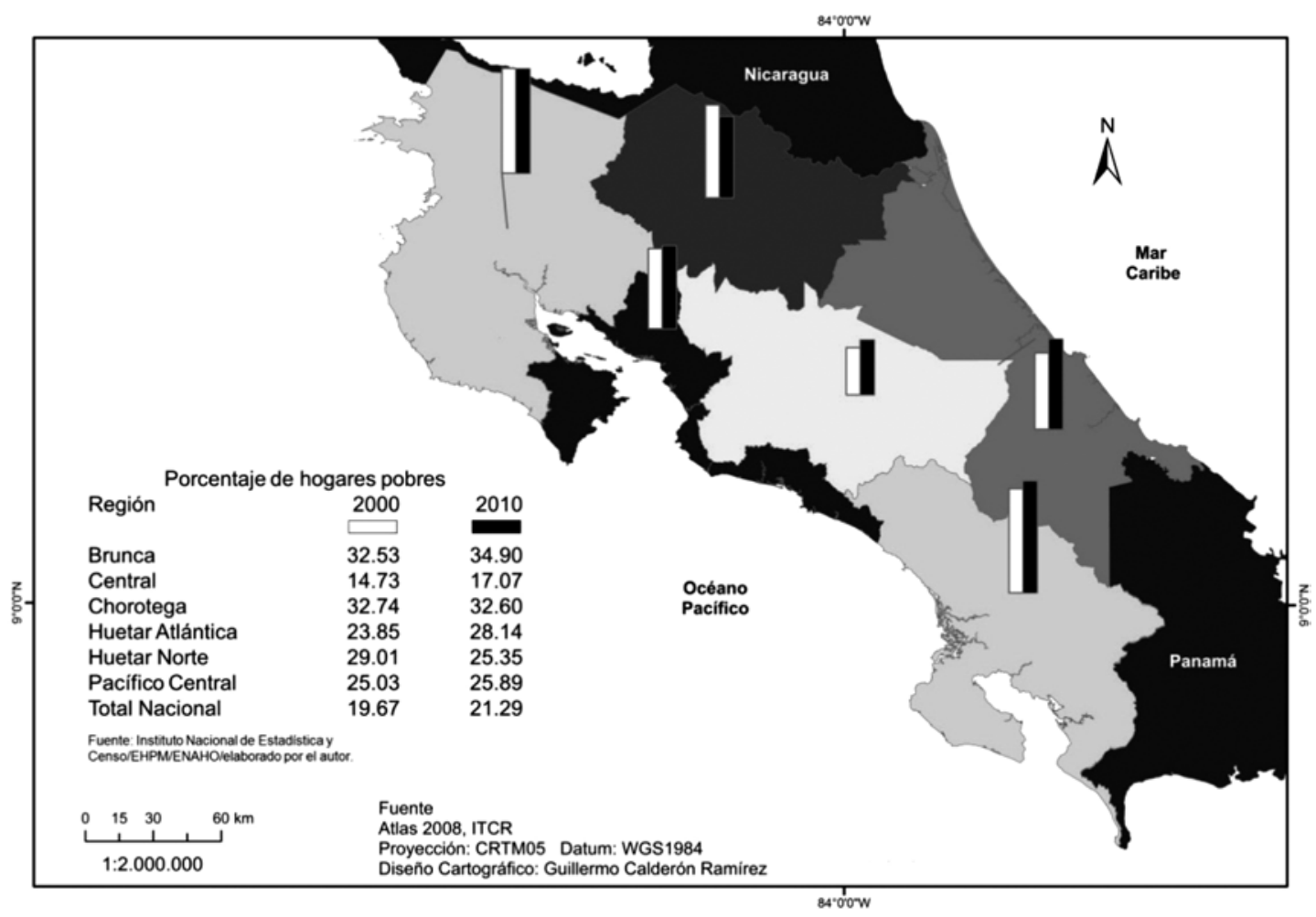

Primer momento: situación del bienestar social antes de los años noventa

En torno de los años 1950 Costa Rica opto por una política social fundamentada en la universalización, en la cual el Estado será el defensor de esos derechos y mediador de los mismos. Además la abolición del ejército en la década de los años cuarenta fortaleció el financiamiento de los recursos económicos direccionados para la salud y educación costarricense.

La iniciativa de universalización de políticas sociales referentes a salud, seguridad social, vivienda, educación, electricidad, agua potable y un desarrollo económico competente fueron en ese entonces claves en el desarrollo humano costarricense, Vega (2010). Estas son algunas de las razones de mayor trascendencia que explican el desarrollo humano costarricense en las últimas décadas en comparación con el resto de América Latina. 


\section{Vivienda}

El tratamiento de una política pública en función de la vivienda costarricense se inicia con la creación del Instituto Nacional de Vivienda y Urbanismo (INVU) el 24 de agosto de 1954 mediante la Ley No.1788, con carácter de institución autónoma. Una vez conformado estableció dos sistemas uno denominado Sistema de Ahorro y Préstamo creado en 1955 mediante el Decreto Ejecutivo n. 1788, destinado a familias con un ingreso medio teniendo como fin el financiamiento para la vivienda con una tasa de interés anual fija de un 9\%. Este préstamo puede ser utilizado por cualquier persona física o jurídica, logrando un préstamo de hasta $\$ 208.764,16$ (este monto es el máximo posible a obtener) lo que le permite a la persona con la capacidad de ahorro obtener su vivienda. El otro sistema fue después de 1986 por medio del Banco Hipotecario de la Vivienda (BANHVI).

Además de la función que desempeña el INVU con el Sistema de Ahorro y Préstamo, la otra función de ayudar a familias de muy bajos recursos económicos depende de la disponibilidad financiera en forma oportuna del BANHVI y de la Dirección de Desarrollo Social y Asignaciones Familiares (DESAF), la cual es la administradora del Fondo de Desarrollo Social y Asignaciones Familiares (FODESAF) siendo este fondo uno de los principales instrumentos de las políticas contra la pobreza.

\section{Salud}

El sistema de protección social costarricense inicia en la década de los años 20 con la creación del Ministerio de Salud, posteriormente se fundan instituciones claves para el desarrollo como la Caja Costarricense de Seguro Social (CCSS) en 1941 y el INVU en 1954. Con ello en la década de 1950 se opta por la universalidad y solidaridad, en donde el país opto por políticas públicas universales tanto en vivienda, salud y educación, teniendo además la intensión de mejorar la renta de su población.

El financiamiento de la política social proviene del FODESAF creado en 1974, financiado por un impuesto del 5\% a las planillas a cargo del patrono y un porcentaje de la recaudación del impuesto de ventas. Por ende Costa Rica inicia con un modelo para apoyar a los sectores en condiciones de pobreza, adelantándose al Banco Mundial el cual para la década de los 1980 propuso en América Latina crear los Fondos de Inversión Social (FIS). 
El panorama de la salud en Costa Rica a mediados de los años cincuenta era caótico, las acciones de salud se basaban en medidas sanitarias colectivas, y la atención a las personas era por medio de consultas privadas, y solo el adinerado podría hacer uso del mismo, (Olazával, 2005). Es entonces que a partir de 1941 se crea la CCSS. Dicha institución llego para quedarse y consolidar el sistema de la salud pública, siendo la CCSS uno de los entes más estables de Costa Rica, hasta la actualidad aportando seguridad e igualdad entre sus usuarios. En síntesis la participación del Estado en el área de salud se dio en la década de 1920 creando el Ministerio de Salud, tomando un nuevo impulso en los años 40 con la creación de la CCSS, y el mayor impacto fue en los años 70 con la universalización del seguro social, (Seligson et al., 2011).

Una de las principales herramientas de la CCSS es el Seguro de Pensiones de Invalidez Vejez y Muerte (Régimen de IVM), permitiendo desde mediados del siglo pasado, que la población trabajadora y sus familias cuenten con cobertura ante la situación de invalidez, vejez o muerte. Este régimen nace en el año 1947 e inicialmente tenía un carácter voluntario, dirigido solo a profesionales, oficinistas del Estado y de instituciones públicas. Ya para el año de 1971, la Junta Directiva de la CCSS aprueba el reglamento del Seguro de Invalidez Vejez y Muerte, para todos los trabajadores directos, pasando a llamarse protección "Régimen Contributivo". Posterior a ello tres años después en 1947 se crea el Régimen no Contributivo de Pensiones, creado por la Ley de Desarrollo Social y Asignaciones Familiares, n. 5662 del 23 de diciembre de 1974, reformada por la Ley No.8783 del 13 de octubre del año 2009. Este Régimen se considera un programa adicional al Seguro de Invalidez, Vejez y Muerte, que administra la CCSS.

Apunta Vega (2010) que entre 1950 y 1980 el marco general del crecimiento económico, la estabilidad política y el nulo gasto militar mostraron dividendos positivos para los costarricenses pasando de un $50 \%$ de la población en condición de pobreza para alrededor de un 30\% respectivamente. Existió un alza en los índices de pobreza a inicio de los años ochenta debido a la crisis a causa del aumento en el precio del petróleo y la deuda externa. Por lo anterior Costa Rica ejecuto un plan de compensación social, fortaleciendo instituciones como la CCSS y el FODESAF, logrando reducir la pobreza en 22,9\% en el año 1994. 


\section{Educación y Renta}

El velar por la condición de pobreza de estudiantes en secundaria le compete al IMAS, esto tiene relación directa con la renta. En 1971 se crea el IMAS mediante la Ley n. 4760 indicando que dicha institución es autónoma con personería jurídica, la cual tiene como finalidad: "formular y ejecutar una política nacional de promoción social y humana de los sectores más débiles de la sociedad costarricense" (Ley 4760, 1971).

Como parte del objetivo de cubrir los gastos de estudiantes en la educación superior, se crea mediante la Ley n. 6041 del mes de febrero de 1977 la Comisión Nacional de Préstamos para la Educación (CONAPE), con carácter semiautónomo del Estado, y en donde el mismo tiene como finalidad principal financiar el proceso académico de estudiantes vinculados a universidades privadas. El CONAPE concede préstamos a costarricenses para realizar sus estudios de educación parauniversitaria y universitaria, dentro o fuera del país, basados en mérito personal y condiciones socioeconómicas de los beneficiarios, los mismos deben ser preferiblemente de zonas rurales.

Según el Estado de la Nación (2013) indica que inicialmente el recurso económico disponible del CONAPE con respecto a la evolución de la educación superior era y provenía de un 5\% de las utilidades anuales netas de todos los bancos comerciales del país, monto que paso a partir del 2011 a ser de un $2 \%$ debido a un cambio en la Ley de Banca para el Desarrollo, y entre otros excedentes de entidades públicas o privadas y donaciones.

\section{Segundo momento: Políticas sociales 1990-2000 \\ Vivienda}

Otro momento importante en el tema de política social en función de la vivienda costarricense se debe a la creación del BANHVI en 1986 mediante la Ley No.7052 teniendo como fin resolver de forma ordenada el problema de habitación de los sectores más pobres. Además del BANHVI se crea el MIVAH por medio de la Ley No.7055, del 22 de diciembre de 1986, Ley General de Presupuestos Ordinarios y Extraordinarios de la República. Siendo el órgano técnico Rector del Gobierno de la República en materia de viviendas y ordenamiento territorial. Desde este Ministerio se formulan políticas y directrices para suplir las necesidades de las distintas condiciones socioeconómicas, con la finalidad de proporcionar acceso a viviendas. Es 
destacable apuntar que aunque este apartado se refiere al segundo momento de políticas sociales (1990-2000), y se discute la creación del BANHVI y el MIVAH debido a que se toma la posibilidad de que su funcionamiento se da en los siguientes años referentes a la década de los años 90.

Sabiendo que en 1954 se crea el INVU con el objetivo de garantizar un acceso a vivienda de las familias con escasos recursos económicos, además del IMAS, Mutuales, entre otras instituciones. Es a partir de esto que se llega a la creación del BANHVI ya que las instituciones anteriormente citadas poseían una descentralización en la cual cada una dictaba sus propias normas y por lo tanto este ente llego para integrar dentro de un mismo sistema con un marco político definido.

El BANHVI es un banco que no atiende a las familias directamente, funciona a través de otras instituciones autorizadas. Esta institución dirige bonos familiares de vivienda estos son donados por el Estado y otorgados a familias de escaso recurso económico y de clase media, familias que presenten alguna condición de riesgo por condiciones ambientales o discapacitados y adultos mayores. El monto máximo a recibir es de $\$ 12.309,21$ el monto a recibir depende de los ingresos de la familia. Junto a esta donación existe un préstamo complementario que esta misma institución otorga. De igual manera el bono depende del ingreso de la familia, siendo un salario mínimo de $\$ 458,01$ con un préstamo de $\$ 12.651,90$ y máximo de $\$ 2.748,19$ y préstamo de $\$ 71.149,19$, con tasa de interés de un $11 \%$ anual.

\section{Salud}

Como ya se explicó el funcionamiento de la salud costarricense direccionada hacia el higiene y el seguro social le compete a la CCSS creada mediante la ley n. 17 del 1 de noviembre de 1941 reformada el 22 de octubre de 1943 siendo autónoma teniendo como ente rector al Ministerio de Salud. La atención primaria de la salud es uno de los principales factores a ser atendidos de forma inmediata, teniendo como objetivo que toda la población tenga alcance al sistema de salud primaria tal como se menciona en la Conferencia Internacional sobre Atención Primaria de Salud:

...la asistencia sanitaria esencial, basada en métodos y tecnologías prácticos, científicamente fundados y socialmente aceptables, puesta al alcance de todos los individuos y familias de la comunidad mediante su plena 
participación y a un coste que la comunidad y el país puedan soportar, en todos y cada una de las etapas de su desarrollo, con un espíritu de autorresponsabilidad y autodeterminación. (Organización Mundial de la Salud, 1978, p. 3-4)

En los años noventa el país fortaleció el sistema de salud pública, ya que desde la década de los 1940 cuando nace la CCSS hasta la década de1990 Costa Rica atendía la salud primaria por medio de los puestos de salud los cuales vinieron a renovarse con los Equipos Básicos de Atención Integral de Salud (EBAIS) actualmente consolidados y en crecimiento tanto de infraestructura como de cobertura poblacional. Señala Olazával (2005) que estos están constituidos por un médico, auxiliar de enfermería, auxiliar de estadística y farmacia y asistentes de atención primaria los cuales aún es la base de la cobertura de salud. Con respecto a la cobertura de los EBAIS estos atienden de 4.000 a 5.000 habitantes, presentando una alta atención en las áreas rurales y urbano-marginales, cubriendo para el año 2002 un $90 \%$ de la población costarricense.

Desde su creación los avances que han tenido los EBAIS en cobertura poblacional son de destacar, afirma la CCSS (2013) en su inventario y análisis de áreas de salud, que para el 2013 un 95\% de la población tiene asignado un EBAIS brindando la atención según el modelo institucional de atención integral, esta se basa en la integralidad, el sujeto de la atención, continuidad, inter y multidisciplinario. Lo anterior no quiere decir que no toda la población costarricense se vea beneficiada con los EBAIS ya que las personas pueden acudir a su centro médico más cercano y serán atendidas.

\section{Educación y renta}

Para los años noventa debido a la crisis que azoto América Latina en la década anterior a esta, la educación sufrió una inestabilidad, además de verse afectado la inversión social en general repercutiendo en las acciones de contribución monetaria de las familias en condición de pobreza costarricense, teniendo en los años siguientes una recuperación vulnerable ante la crisis económica. 


\section{Tercer momento: Políticas sociales 2000-2010 \\ Vivienda}

El fortalecimiento en el tema de vivienda para familias en condición socioeconómica media o pobre, fue acentuado antes del siglo XXI. Como se mencionó anteriormente Costa Rica realizó avances importantes en el tema de vivienda tanto con el INVU como con el BANHVI y que prevalecen hasta la actualidad. Es interesante destacar que en esta investigación no se obtiene los datos referentes a los beneficiarios de esta política o cual es la ejecución de la misma, más se intenta evaluar la política pública para vivienda, debido a que como se señala en los ítems anteriores se tienen tres instituciones distintas (INVU, BANHVI, MIVAH) que trabajan para que familias en condición media o pobre logren acceso a una vivienda.

\section{Salud}

Luego de inicios de la primera década del siglo XXI, mediante la Ley de Protección al Trabajador del Sistema Nacional de Pensiones, el Seguro de Pensiones de Invalidez, Vejez y Muerte se convierte en un sistema básico y obligatorio; además de la obligatoriedad de la afiliación de los trabajadores independientes sean asalariados o no.

\section{Educación y Renta}

Para mejorar la calidad de vida de los estudiantes de secundaria nace el programa AVANCEMOS mediante el Decreto Ejecutivo $N^{\circ} 33154$ del 2006 con el gobierno de Oscar Arias Sánchez. Este según el decreto ejecutivo $\mathrm{N}^{\mathrm{o}} 34786-\mathrm{MP}-\mathrm{S}-\mathrm{MEP}$ tiene como población meta familias integradas por adolescentes y jóvenes de ambos sexos con edad entre los 12 y los 25 años, que presenten condición de pobreza, vulnerabilidad social y exclusión, que necesiten apoyo económico para lograr mantenerse en la educación secundaria. Este programa es destinado a familias en condición de pobreza, fortaleciendo el sistema educativo, siendo un complemento en la renta familiar para que los padres o encargados mantengan a los jóvenes en la enseñanza media.

El inicio de AVANCEMOS se dio como fase piloto, ejecutado por el IMAS y FONABE. No obstante a partir del 2009 se logró definir una única unidad ejecutora el IMAS, señalada en el decreto ejecutivo 34786-MP-S-MEP, trayendo una simplificación en el manejo de la 
población meta. El financiamiento al igual que el programa del INVU es por medio del FODESAF y de recursos del presupuesto nacional, recursos propios y donaciones.

El seguimiento o monitoreo de la beca establecida se desarrolla por el método Sistema de Población Objetivo (SIPO) la cual utiliza como herramienta la Ficha de Información social (FIs) está contiene 64 variables enfocadas a los núcleos familiares. Dicho método clasifica las familias por nivel de prioridad mediante cuatro categorías de puntaje: Grupo 1: los más pobres; Grupo 2: pobreza moderada; Grupo 3: personas con nivel menor de pobreza; Grupo 4: resto de la población beneficiada. El monto de transferencia es escalonado dependiendo del nivel educativo, reiterando que es un complemento con respecto a la renta de las familias. (Tabla 1).

Tabla 1. Costa Rica: monto de transferencia según año educativo del beneficiario (dólares)

\begin{tabular}{|c|c|c|c|}
\hline Nivel Educativo & Mensualidad* & $\begin{array}{c}\text { Monto Anual } \\
\text { Mínimo* }\end{array}$ & $\begin{array}{c}\text { Monto Anual } \\
\text { Máximo* }\end{array}$ \\
\hline $7^{\mathbf{0}}$ & 29,54 & 354,51 & 470,70 \\
\hline $8^{\mathbf{0}}$ & 39,39 & 472,67 & 588,87 \\
\hline $9^{\mathbf{o}}$ & 49,24 & 590,84 & 825,21 \\
\hline $10^{\mathbf{0}}$ & 68,93 & 827,18 & $1.061,55$ \\
\hline $11^{\mathbf{o}}$ & 88,63 & $1.063,52$ & $1.179,71$ \\
\hline $12^{\mathbf{o}}$ & 98,47 & $1.181,68$ & $1.416,05$ \\
\hline
\end{tabular}

* Tipo de cambio del dólar referente al año 2012 según el Banco Central de Costa Rica Fuente: Decreto Ejecutivo No 34210 ; elaborado por el autor.

La función de aporte de becas a educación primaria y no menos importante la situación de los estudiantes de post secundaria en la cual el FONABE y el CONAPE son las encargadas de esta población, siendo esta última financiadora por medio de préstamos. Las mismas son instituciones del Estado que trabajan de manera separada pero tienen como objetivo mejorar la educación nacional.

El funcionamiento en la educación superior costarricense tiene como base la creación del FONABE en 1997 la cual conto con la aprobación de los diputados incorporándose el Ministerio de Educación Pública (MEP) del que depende como órgano adscrito mediante la Ley n. 7658 del 27 de 
febrero del 1997, años después en el 2004 se amplía la cobertura de becas para estudiantes de post secundaria. Además en ese mismo año se crea una nueva modificación al Fondo el cual incluye a los extranjeros en igualdad de condiciones de selección.

El MEP indica en el "Plan operativo institucional y presupuesto ordinario 2012" que la beca por condición socioeconómica referente a primaria contiene a la población con rango de edad entre 7 y 12 años, o mayor si presentara rezago escolar. Este aporte mensual es de $\$ 33,48$ con el fin de financiar parte de los costos educativos de estudiantes que se encuentren en condición de pobreza extrema, pobreza o vulnerabilidad.

El FONABE es una institución sin fines de lucro que tiene como objetivo brindar ayuda a estudiantes de familias con escasos recursos económicos para que curse y concluyan exitosamente el proceso educativo. En el "Plan operativo institucional y presupuesto ordinario 2012" se sugiere que por medio del programa de becas post secundaria se realiza el esfuerzo de cubrir la población con condición de vulnerabilidad económica y educativa de Colegios Universitarios o parauniversitarios, universidades públicas y privadas este último con la condición de ser reconocidas por el Consejo Nacional de Educación Superior Privada (CONESUP).

Ahora bien la forma de ejecución del préstamo según el CONAPE en sus requisitos y garantías se señala una serie de requisitos y garantías en las cuales el solicitante debe presentar documentación de él mismo, del núcleo familiar, del colegio de procedencia, de la universidad en la que se encuentra estudiando, y asociado a ello otros requisitos para el caso de posgrados tanto fuera como dentro del país.

Al tener requisitos también cuentan con garantías divididas en fiduciaria o hipotecaria; la primera funciona para montos desde $\$ 5.908,42$ hasta $\$ 17.725,26$ y la segunda con préstamos mayores a $\$ 17.725,26$ hasta $\$ 59.084,19$ con una tasa de interés de un $8 \%$. Este interés es anual sobre saldos, variable y ajustable según los términos del artículo 497 del Código de Comercio el cual indica que:

Se denomina interés convencional el que convenga las partes, el cual podrá ser fijo o variable. Si se tratare de interés variable, para determinar la variación podrán pactarse tasas de referencias nacionales o internacionales o índices, siempre que sean objetivos y de conocimiento público... (Ley 3284, 1964). 
El pago del préstamo es a partir de concluidos los estudios, no obstante el prestatario puede solicitar un período de gracia el cual es de seis meses después de concluido su etapa de estudio. Si el estudiante reprueba un curso el CONAPE no las financia nuevamente, por ende el prestatario deberá cubrir dicho gasto. Si el hecho de reprobar alguna materia repercute con la extensión del plan de estudio el mismo debe presentarse a CONAPE y ajustar dicho programa. Además de que todo estudiante debe matricular el bloque completo de créditos según el plan de estudio que este cursando.

El funcionamiento del CONAPE ha tenido resultados positivos se menciona en el Programa Estado de la Nación en Desarrollo Humano Sostenible (2013) que su informe Estado de la Educación en sus 36 años ha colocado 89.927 préstamos los cuales han sido de importancia para estudiantes de universidades privadas, siendo que entre el 2008 y 2011 el 70,1\% fue dirigida a zonas con menor desarrollo relativo. Todo lo anterior lleva a re pensar en cómo se están manejando las políticas en función del bienestar costarricense, por lo tanto a continuación se señalan algunas consideraciones.

\section{Consideraciones finales}

El estudio busco profundizar sobre conceptos importantes para analizar la pobreza y se mostró el ejemplo costarricense. Además el trabajo incentivó en detallar la relevancia de la geografía en investigaciones científicas, subrayando la importancia de esta ciencia en temas sociales los cuales son desarrollados en una dimensión espacial con características particulares, en donde coexisten efectos territoriales provenientes de los conflictos políticos. Se acentúa acerca del papel del geógrafo como crítico con herramientas y capacidad necesaria para contribuir al debate, resaltando su representatividad en problemas sociales.

Se debe tener presente que no existe metodología capaz de responder a todos los cuestionamientos que surjan de un tema dado, no obstante con el proceso metodológico aquí propuesto se logra cubrir los objetivos planteados, diagnosticando la pobreza costarricense e interpretando como las políticas públicas contribuyendo en dicho proceso. A través de dicha metodología la cual inicia con la política como primer elemento de análisis en el que el Estado-Nación tiene como reto mejorar el bienestar social y además es mediador de las políticas públicas, analizadas a través de los programas sociales (vivienda, salud, educación y renta). Dicho proceso 
es desarrollado considerando la geografía política, en donde intervienen agentes sociales, económicos y políticos.

El abordaje de la pobreza costarricense ha sido un tema de gran discusión debido a las distintas tesis del posible estancamiento que se viene dando desde las dos últimas décadas (1990-2010), por lo tanto era un desafío descubrir la existencia o no de dicha situación y como las políticas públicas contribuyen en la disminución de la pobreza. Parece existir una inmovilidad de las política juntos a sus programas de acción lo cual a causa de las transformaciones de la población deberían de estar en una transición paralela a los cambios de la sociedad. Por lo tanto no solo la cobertura es necesaria sino también la calidad de los servicios, mejor preparación del personal y equipos tecnológicos.

La trayectoria que han tenido políticas relacionadas a la vivienda y salud iniciada en la década de los años 50 en el caso del tratamiento de las viviendas y en la década de los años 20 para la salud han sido incluso ejemplos en América Latina por su historia y capacidad nacional. Existe una descentralización en la ejecución de la política por ejemplo en el tema de la vivienda instituciones involucradas como el INVU, BANHVI o MIVAH trabajan para un mismo fin pero de manera aislada. La salud pública ha tenido su fortaleza en la Caja Costarricense de Seguro Social la cual tomo la dirección y dicto las pautas como líder en la seguridad de la salud costarricense, por lo tanto esta centralización de funciones ha beneficiado a la población.

El motivo de evaluar la educación junto a la renta se debe a que en algunos de los casos los escolares para recibir un aporte monetario, la familia debe encontrarse en condición social vulnerable. Aunque instituciones y programas que velan por la educación creadas desde la década de los años 70 consiguieron elevar las tasas de alfabetización, ya para los años 90 se evidencia un estancamiento en el nivel de educación principalmente en la secundaria consecuencia de la crisis de la década de los 80 aplacando el ritmo con el que se venía creciendo. No obstante una de los principales motivos en la evolución de la educación secundaria parece ser causada por la mala administración y lenta ejecución de algunos programas como AVANCEMOS creado en el año 2006. En resumen a excepción del tema de la salud existe una descentralización en la ejecución de los programas sociales que atienden la política pública. Esto podría ser atribuido a una necesaria revisión de las políticas y un manejo adecuado de las mismas. 


\section{Referencias}

Abranches, S. (1998). Política Social e Combate à Pobreza: A teoria da prática. En: S. Abranches, W. d. Santos, \& M. Coimbra, Política Social e Combate à Pobreza (pp. 9-31). Rio de Janeiro: Jorge Zahar. Arendt, H. (2003). O que é politica? (4 ed.). Rio de Janeiro: Bertrand Brasil.

Azevedo, J. M. (1997). A Educação como Política Pública. Campinas: SP: Autores Associados.

Bobbio, N. (1987). Estado, poder e governo (6 ${ }^{\mathrm{a}}$ ed.). En Estado, governo e sociedade; por uma teoria geral da política (pp.53-133). Rio de Janeiro: Paz e Terra.

Britto, L. N. (1986). Política e Espaço Regional. Sao Paulo: Nobel.

Castro, I. (2005). Relações entre território e conflito: o campo da geografia política. En: Castro, I, Geografia e política. Território, escalas de ação e instituições (pp. 44-45). Rio de Janeiro: Bertrand Brasil.

Caja Costarricense de Seguro Social. (2013). Gerencia Médica. Dirección Proyección de Servicios de salud. Inventario y análisis de salud, sectores, ebais, sedes y puestos de visita periódica en el ámbito nacional. Demo, P. (1996). Política Social e Educação e Cidadania. (2 ed.) Campinas, SP: Papirus.

Enciclopédia do mundo contemporâneo. (1999). São Paulo: Publifolha; Rio de Janeiro: Editora Terceiro Milênio

Font, J.N. \& Rufi, J.V. (2001). Geopolítica, identidad y globalización. (1. ed.) Barcelona: Editorial Ariel, S.A.

Gomes, P, C, da C. (2000). O Conceito de região e sua discussão. En: Castro, I.E de et al. (Org.) Geografia: conceitos e temas (2 ed., pp.4976.). Rio de Janeiro: Bertrand Brasil.

Johnston, R. J., Gregory, D. \& Smith, D. M. (2000). Diccionario Akal de Geografía Humana. Barcelona: Akal.

Lencioni, S. (2003). Região e Geografia. São Paulo. Editora da Universidade de São Paulo.

Novaes, A. (2003). Invenção e crise do Estado-nação. En [Org.] A crise do Estado-nação (pp.9-22). Rio de Janeiro: Civilização Brasileira.

Olazával. H.V de. (2005). Atención primaria de salud y Salud para Todos: Costa Rica y Centroamérica. Escenarios, participación, desafíos 
siglo XXI.MINSA. CCSS.OPS.OMS. Atención Primaria de Salud en Costa Rica: 25 años después de Alma Ata.

Organización Mundial de La Salud. Conferencia Internacional sobre Atención Primaria. Alma-Ata. URSS, 1978. Disponible en: <http:// whqlibdoc.who.int/publications/9243541358.pdf>.

Programa Estado de la Nación en Desarrollo Humano Sostenible. (2013).

Cap. 1. Cuarto Informe Estado de la Educación/PEN. - 4 ed. - San José C.R: Editorama.

Programa Estado de la Nación. Estado de la Educación, 2013. Capítulo 4. La Evolución de la Educación Superior. Disponible en: $<$ http://www. estadonacion.or.cr/index.php/estadoeducacion/educacioninformeultimo $>$. Consultado el: 2 de setiembre 2013.

Rocha, S. (2006). Pobreza no Brasil: a final, de que se trata? (3. ed) Rio de Janeiro: FGV

Santos, M. (1988). Metamorfoses do Espaço Habitado. Sao Paulo. Hucitec. Santos, M. (1978). Pobreza Urbana. São Paulo: Hucitec.

Seligson, M.A., Franzoni, J.M. \& Solórzano, J.D.T. (2011). Reducción de la pobreza en Costa Rica: El impacto de las políticas públicas. En Solórzano, J.D.T. (Compilador). La Pobreza en Costa Rica: estudios del Instituto de Investigaciones de Ciencias Económicas (1 ed., pp. 215-324.) San José, Costa Rica.

Souza, C. (2006). Políticas Públicas: uma revisão da literatura. Sociologias. Porto Alegre, 16, 20-45.

Taylor, P. \& Flint, C. (2002). Geografía Política - Economía Mundo, Estado Nación y Localidad. (2 ed.) Madrid: Trama Editorial.

TrigaL, L.L. \& Pozo, P.B. del. (1999). Geografía Política. Madrid: Cátedra. Vega, I, R. (2010). Costa Rica: pobreza y desigualdad como determinantes de la salud en los últimos 20 años. En: Universidad de Costa Rica. Escuela de Salud Pública. (Org.) La salud pública en Costa Rica: Estado Actual, Retos y Perspectivas. (pp.149-168). San José

Wolff, F. (2003). Invenção e crise do Estado-nação. En: Novaes, A. [Org.] A crise do Estado-nação. Rio de Janeiro: Civilização Brasileira. 